\title{
PERFORMANCE DRIVEN GLOBAL ROUTING FOR STANDARD CELL DESIGN
}

\author{
Jason Cong Patrick H. Madden \\ UCLA Computer Science Department* \\ 4711 Boelter Hall \\ Los Angeles, California 90095 \\ \{cong, pickle\}@cs.ucla.edu
}

\begin{abstract}
Advances in fabrication technology have resulted in a continual shrinkage of device dimensions. This has resulted in smaller device delays, greater resistance along interconnect wires, and a greater impact of interconnect on total system performance. These changes have driven a considerable number of studies on single-net interconnect optimization, but relatively little work has been done to integrate the results on single-net optimization with the problem of global routing and interconnect optimization for the entire circuit. In this paper, we present the DECIMATE global router for performance driven standard cell design. The router applies both interconnect topology optimization and variable-width wire sizing optimization results to the global routing problem, while maintaining routing areas that are comparable with TimberWolf Systems' well-known commercial global router. Optimal selection of interconnection structures is shown to be an NP-Hard problem; we provide a simple heuristic for the problem, and show that it is effective with experiments on industry benchmarks. Under the Elmore delay model, our global router produces as much as a $35 \%$ reduction in critical path delay over TimberWolf Systems' global router, while path length reductions are as large as $52 \%$. Circuit area optimization is performed taking into account variably-sized wires, fixed routing topologies, and pre-existing obstacles; an improved cost function obtains as much as an $11.6 \%$ reduction in channel density over the result in $[16]$.
\end{abstract}

\section{INTRODUCTION}

With the advent of deep submicron design, a number of process parameters have changed, resulting in an increasing importance for interconnect optimization. Interconnect delay can now consume from $50 \%$ to $70 \%$ of the clock cycle in many cases [3]; reduction in interconnect delay will have a significant impact on the overall performance of the circuit.

${ }^{*}$ This work is partially supported by DARPA/ITO under Contract J-FBI-93-112 and NSF under Young Investigator Award MIP-9357582.
To address these new design parameters, a number of approaches to single net interconnect topology optimization have been proposed, such as bounded-radius bounded-cost trees[10], AHHK trees[1], maximum performance trees[9], A-trees[14], IDW/CFD trees[21], SORT and SERT trees [4], and P-Trees[28]. These methods consider both the traditional concern of low total tree length, and also the path length or Elmore delay between the source node and the timing-critical sink nodes. Many of these algorithms have been surveyed in [24] and [12].

In addition to topology optimization, sizing of interconnect wires has also been shown effective for delay reduction $[13,15,27,33,8,31]$. Traditionally, minimum width wires were used for most connections; for high performance submicron design, however, this may be inappropriate.

The global routing problem (with area minimization objectives) is NP-hard in general, and has been studied by a number of researchers.

A hierarchical decomposition of the global routing problem has been used with some success $[18,6]$, dividing the core area into progressively smaller regions. Simulated annealing has also been applied to the global routing problem [26], where both net topologies and cell positions may be affected. Linear programming methods have been used to select the assignment of segments in $[2,22]$, in order to minimize the maximum density across a channel. Related to the LP methods are those based on network flow or multicommodity flow models $[30,36,7,34]$. In [20], the authors use path-based timing constraints and utilize the features specific to bipolar design to optimize both the delay and area of the global routing result.

The global router described in $[16,17]$ provides the basis for the work in this paper. It involves two phases, a constructive step in which feedthroughs are inserted, and an iterative deletion step which first constructs redundant connection graphs for each net, and then removes redundant edges to minimize overall channel density.

Most of the works on interconnect optimization deal with only single net optimization, and do not address the issues of how to integrate the optimization techniques into global routing. On the other hand, most existing global routing approaches fail to address global delay minimization for deep submicron design. This paper presents the DECIMATE global router, which addresses both of these problems, and offers the following features.

- Through the use of high performance interconnect topologies, the router addresses global path delay concerns for timing driven circuit design. We apply the topology algorithms of $[14,23,5]$ and the wiresizing work of $[15,13]$ in our global router. 
- Area optimization takes into account both the timingcritical and non-timing-critical nets, as well as variable width routing and pre-existing congestion. The router also avoids the rip-up and re-route approach common in global routing, obtaining low area solutions directly. Area results are competitive with the well respected TimberWolf Systems' global router on widely available industry benchmarks, showing that interconnect optimization can be considered with little or no sacrifice in circuit area.

\section{PROBLEM FORMULATION}

The standard cell model has been well studied, and is widely used for ASIC designs. Standard cell design allows the construction of relatively high performance circuits with moderately low design effort.

In standard cell design, a circuit is composed of a set of logic cells; each cell contains a number of connection points, commonly called pins or ports. There may be equivalent pins, which allow connection at multiple locations on a cell (these points are electrically equivalent). A net is a set of pins which must be interconnected. Cells are arranged into horizontal rows. Interconnection takes place in horizontal channels between the rows, using channel segments. In two layer design, one metal layer is used for the horizontal channel segments, while the second metal layer is used for vertical connections between the cells and the channel segments. If three or more layers are available, over the cell routing may be used. This paper assumes a two-layer model, and discusses extensions to three or more layers in Section 5. Through the insertion of feedthroughs in the row or the utilization of built in feedthroughs, inter-channel connectivity can be obtained.

The traditional global routing problem is to determine the connection pattern for each net to minimize the overall routing area. The density of a channel corresponds to the maximum number of horizontal segments passing any point within the channel. The density metric has been found to be an accurate estimate of the routing area required by the channel; the extension of this metric to variably sized interconnect wires is straightforward. The area required to route a circuit is a function of the longest cell row and the density across all channels (with consideration of variable wire widths).

In high performance design, we are concerned not only with circuit area, but also with signal delay and operating rates. Signal delay through a complex VLSI circuit involves a series of interconnect nets and logic gates. To maximize device operating rates, delay along the critical path (the longest delay path) through the circuit must be minimized. Note that determination of the critical path through a circuit is an NP-hard problem in general (due to the false path problem). For the purposes of this discussion, we consider the static critical paths, which are the maximum delay paths from primary inputs or flip-flop outputs to primary outputs or flip-flop inputs, where the delay of a path is the sum of gate delays and interconnect delays along the path. If we wish to perform delay optimization, we must select a subset of nets for interconnect optimization, while avoiding large penalties in terms of circuit area.

As an example, a pair of possible interconnect topologies are shown in Figure 1. The first example shows an interconnect solution for a timing critical net minimized for total wire length (a traditional global routing objective). While wire length is low, delay from the driver (at the top of the circuit) to the critical sink (towards the left side of the circuit) is high. The second example shows a routing (in this case, an A-Tree [14]) which provides shortest paths from the driver to the critical sinks, and also provides reduced delay.

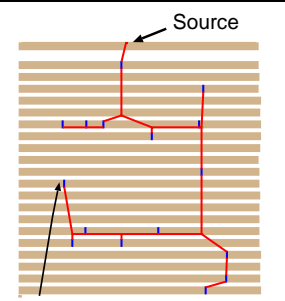

Critical sink

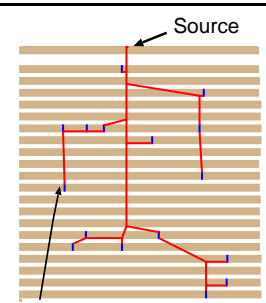

Critical sink
Figure 1. An area-minimizing interconnect structure for the net $\mathrm{B3}$ in the MCNC example STRUCT, and a delay minimizing structure. While a minimum-length Steiner tree is appropriate for area minimization, it may result in high delay for deep submicron design.

\section{THE DECIMATE GLOBAL ROUTER}

The goal of a global router is to specify interconnect structures (topologies and wire sizes) and feedthrough locations for signal nets. For the solution to be acceptable, it must allow for low core area, and also low critical path delay.

With this in mind, we partition the global routing problem into three subproblems, and then solve these subproblems efficiently. The global router first generates interconnect structures appropriate for each net, then inserts and assigns feedthroughs to each net, and finally selects channel segments for net connections.

\subsection{Interconnect Structure Generation and Selec- tion}

For submicron design, area-minimum interconnect does not necessarily result in low delay, and low delay interconnect may not be area-minimum. For high performance routing, we are faced with the problem of selecting a subset of nets to route with low-delay structures.

We define the Interconnect Selection Problem as follows: Each net $n_{i} \in N$ has a set of possible interconnect structures $I_{i, a}, I_{i, b}, \ldots I_{i, k}$, with costs $C_{i, a}, C_{i, b}, \ldots, C_{i, k}$. The source-to-sink delays for each net are computed by an appropriate delay model. The Interconnect Selection Problem is to select an interconnect structure for each net which obtains minimum circuit delay subject to a cost constraint.

For example, we might consider minimum-length spanning trees (MSTs), minimum-length Steiner trees, or any of the high-performance interconnect structures mentioned in the introduction, with or without driver or wire sizing, for each net. The cost of an interconnect structure might be measured as the total wire length, the impact of the structure on circuit area, or by some other value. While there may be an infinite number of possible interconnect structures, and evaluation of structure cost may be quite complex, we will restrict our consideration to a tractable set of candidate structures, and will use wire length, linear delay, or Elmore delay as our objective (more accurate delay models may be used as well). While the number of structures we consider for each net may be small, and the 
cost functions are well defined, the problem is still quite difficult.

\section{Fact 1 The Interconnect Selection Problem is NP-Hard.}

Proof: Consider any instance of the well known NP-Hard Knapsack problem [29], and assume that the costs and delays of different interconnect structures in an instance of an Interconnect Selection Problem can be assigned independently. We can construct a chain of inverters, and assign each interconnection a cost and gain for an "optimized" structure that matches the weight and gain of an item from the Knapsack problem. Solution of the Interconnect Selection problem also solves the Knapsack problem.

The problem as defined above is considerably simpler than the practical problem. We assume here that costs and delays of interconnects are independent, although this is not the case in practice. Under complex delay models, the choice of an interconnect structure may affect the delays to different pins in the same net in different ways. Thus, even if we do not constrain the overall cost, the problem of delay minimization can still be quite difficult.

In our global router, we support minimum spanning trees, low area topologies (using the algorithms of [23] and [5]), high-performance topologies (using the algorithm from [14]), and also variable-width interconnect sizing (using the optimal wire-sizing algorithms from $[13,15]$ ). Our heuristic for the Interconnect Selection Problem initially implements minimum spanning trees for all nets (the motivation for this is shown in Sections 3.2 and 4 ), and then evaluates the delay of the circuit. If delay constraints are not met, nets are iteratively selected for delay improvement. We implement a simple greedy algorithm for net selection. For a net $n$, with driver $s$ and critical sink $t$, with path length between the driver and sink in routing tree $T$ as $d_{T}(s, t)$, and Manhattan distance as $d(s, t)$, the gain of the net is defined as $d_{T}(s, t)-d(s, t)$. The highest gain net (with total tree length used to break ties) is selected and then converted to a high performance topology, and may also be sized. The process continues until delay objectives are met, no gain can be found, or improvement falls below a userdetermined threshold. Specific interconnect structures for nets may also be selected manually, allowing experienced designers to perform sophisticated delay analysis and optimization.

A large gain indicates that the path length between $s$ and $t$ can be improved significantly through topology optimization. This may result in a reduction in path interconnect resistance, and also a reduction in delay. Larger trees have a greater chance of improvement through wire sizing, so tree size is used to break ties.

In brief, our approach to interconnect generation and selection is as follows.

- Construct interconnect topologies for all nets.

- Select the highest gain net on a critical path.

- Apply topology optimization and wire sizing optimization to compute a high-performance interconnect structure.

- Recalculate critical paths.

- Repeat selection and optimization until improvement falls below a specified threshold, or delay bounds are met.

While this heuristic is exceedingly simple, it is shown to be effective in Section 4, particularly in circuits with large nets (where simple interconnect topologies may differ greatly from high performance topologies). For other

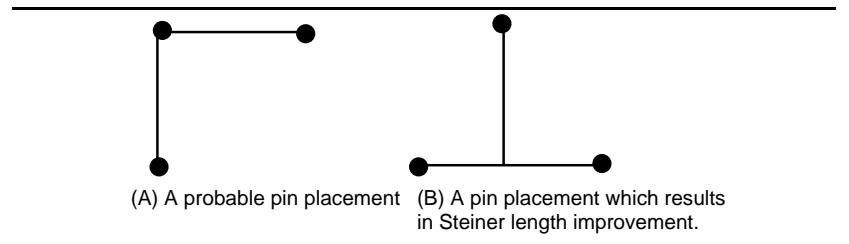

Figure 2. A likely positioning of pins for a three pin net $(A)$, and a positioning of three points which leads to an improvement with a Steiner tree heuristic (B).

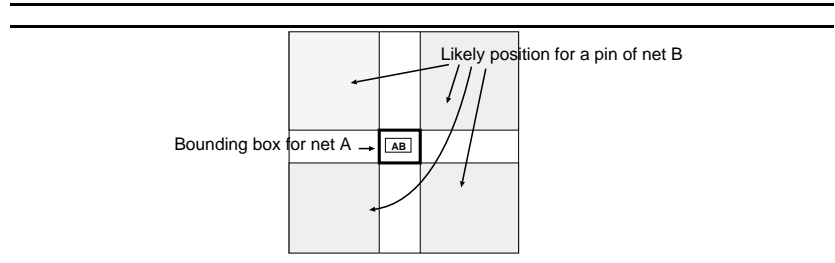

Figure 3. The bounding box for a net $A$, and the likely position for another pin for some net $B$.

circuits, a hill-climbing heuristic with a greater variety of interconnect structures, or manual selection of interconnect by an experienced designer, may be more appropriate.

\subsection{MSTs vs. Steiner Trees}

As mentioned above, we generally implement minimum spanning trees for net interconnection, even though we support a number of Steiner heuristics as part of the global router. While Steiner heuristics can obtain tree length improvements of $10 \%$ or more on random examples, we found improvements to be less than $2.1 \%$ with standard cell designs (see Table 1 in Section 4).

First, note that a large percentage of nets contain only two pins, eliminating any possibility for Steiner tree improvement. For nets with three or more pins, we find that the pin positioning shown in Figure $2 \mathrm{~A}$ is representative of standard cell interconnect problems, and that Figure 2B (which allows tree length reduction when Steiner points are considered) is relatively uncommon.

We consider the lack of improvement for a Steiner tree heuristics on actual placements to be a side-effect of a common placement cost metric. A typical objective function is to minimize the sum of the perimeters (or half the perimiters) of the bounding boxes for all nets in a circuit. Consider the case shown in Figure 3; for a cell which contains two pins, one for net $A$, and one for net $B$, there are certain locations within the bounding box for net $A$ that minimize cost with respect to net $B$. In particular, if net $B$ has one other pin, it is likely to be in one of the four shaded regions shown (as the area of the shaded region is substantially larger than the non-shaded region). We can expect that an optimization process will attempt to move the cell towards one of the four shaded regions; if expansion of the bounding box for $A$ is to be avoided, the desired position will be at the corner of the bounding box. Therefore, if bounding box perimeter is a placement objective, we can expect pins to be placed towards the corners of their net bounding boxes, and tree length reductions through the insertion of Steiner points will be small. 


\subsection{Support for Wire Sizing Optimization}

As mentioned in the introduction, we support variably sized interconnect in our global router. If wire sizing is to be performed, we utilize the optimal sizing algorithms of $[15$, 13] to determine sizes for the edges of nets that are selected for topology optimization. When the net is embedded for the Iterative Deletion process (described in Section 3.5), the channel segment widths are obtained from the width of the topology edges.

To accurately model the delay effects of sized interconnect wires, we model wire capacitance as the sum of area capacitance (which changes with wire width), and the fringing capacitance (which is fixed). Wire resistance is inversely related to the wire width.

The effects of wire width on circuit area are as follows. Each interconnect segment is modeled as a wire with additional spacing (to meet design rule constraints). When a wire is sized, the spacing does not increase. Therefore, a wire that is twice the minimum width takes less routing area than two parallel minimum width segments.

In our current implementation, wire width is uniform within each segment; it was shown in [11] that when the length of segments were small, solutions had near optimal performance. We can easily use the bundled refinement algorithm of [11] to vary wire width within a segment if necessary.

\subsection{Feedthrough Assignment}

If an edge of a net interconnect topology spans one or more standard cell rows in two-layer design, feedthroughs in those rows must be inserted or assigned to the net.

While a number of approaches to single-row feedthrough assignment have been considered, we find that a lack of a global perspective on the problem can seriously degrade solutions. To capture the global nature of the problem, the DECIMATE global router constructs lists of all feedthrough requirements and resources across all rows. Assignment is performed in a greedy manner, assigning a single feedthrough to an edge at each step by increasing order of cost (even though the edge may require more than one feedthrough). The assignment is improved by a greedy pairwise swapping phase.

During our research, we have explored two alternative feedthrough assignment approaches. The first, similar to that of [16], assigns feedthroughs to each interconnect edge based on an edge ordering. In any ordering, edges which receive assignments early in the process obtain high quality assignments, while edges considered later receive lower quality assignments (resulting in significant horizontal jogs). This problem persists under a variety of edge orderings. Horizontal jogs of the feedthrough assignments resulted in a substantial increase in total channel density. On average, our global feed-at-a-time approach resulted in an average $7.1 \%$ decrease in total channel density over the edge-byedge approach.

A second approach based on a bipartite matching between a single row of feedthrough resources, and the requirements of all edges crossing the row, was also explored. Results from this approach were also inferior. The quality of results from this approach suffered from a lack of consideration of multiple row feedthrough requirements. In our experiments, we observed that roughly half of the edges which required feedthroughs needed more than one. Any single-row based assignment must be performed without fixed locations for many of the feedthroughs required; an assignment which minimizes an objective function for one row may result in a very difficult problem for another row, and global solution quality suffers. Bipartite matching is also computationally expensive and scales poorly to large examples.

\subsection{Iterative Deletion}

Selection of high-performance interconnect structures will result in many critical nets having fixed routing topologies, with variable width routing being possible. The pins connected to these nets, and the cells to which they belong, also have fixed positions: shifting of these cells may change the net delay, requiring new interconnect structures to be determined. As high-performance interconnect is not necessarily area minimizing, we are interested in finding interconnect structures for the non-critical nets which are compatible with the fixed high-performance structures.

We approach this problem through the application of the iterative deletion method. This method was first introduced in [16], and is a generalization of the solution to the switchable segment problem $[37,35]$.

\subsubsection{The Switchable Segment Problem}

The switchable segment problem involves determination of the orientation of edges connecting pairs of pins in the same row (referred to as 2-pin linear nets). Generally, a pair of pins in a row can be connected with a segment in either the channel above the row, or the channel below. An example of a 3 -net switchable segment problem is shown in Figure 4.

Solving the problem entails finding the orientation (either above the row or below) of each segment for each pair of pins which minimizes the total channel density. This problem was shown to be NP-hard, and a heuristic no more than 1.5 times optimal was presented in [37].

Many global routing approaches attempt to minimize the total density across all channels by switching segments up and down iteratively. In [35], experiments indicated that $38 \%$ of segments were switchable.

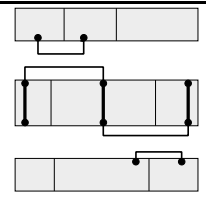

4 routing tracks

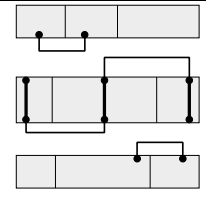

2 routing tracks
Figure 4. Each 2-pin net in the same cell row can be routed using one of the two switchable segments. The selection of switchable segments can have a major impact on the total density, and thus affect the circuit area.

\subsubsection{Iterative Deletion}

Instead of optimizing only simple 2-pin linear nets, we attempt to find an optimal subset of edges from the "simplified net connection graph," introduced in [16].

For each net, the simplified net connection graph is constructed by creating edges between the adjacent pins of a net in each channel. This graph may contain redundant edges, which will be iteratively removed based on their relative costs. An example of such a graph is shown in Figure 5. A simple biconnectivity algorithm can be used to determine which edges are redundant (can be removed), and which are required for connectivity of the net. It was shown 
in [16] that the number of edges in the simplified net connection graph, and the number of edges that must be removed, are linear with the number of pins in the circuit, and that this formulation contains a minimum-density solution.

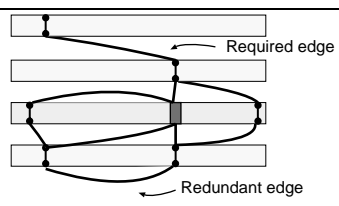

Figure 5. The simplified net connection graph contains edges between adjacent pins in a channel. The final net topology is a subset of these edges.

The DECIMATE global router applies the iterative deletion method in the following way. First, it constructs the simplified net connection graph for each non-critical net. We use $S$ to denote the set of all edges from all simplified net connection graphs. Edges are then removed one-by-one from $S$ by selecting the highest cost redundant edge, and then recalculating costs for the remaining edges. Deletion continues until all redundant edges are removed. Details of the cost functions for edge selection are given in Section 3.5.3.

Since we start with all possible connections, the algorithm has a global view of the congestion distribution. This allows for effective area and congestion minimization without going through a lengthy, usually ad hoc, process of rip-up and re-route.

Note that the iterative deletion method is applied only to nets which are not timing critical; as the iterative deletion process may modify a the topology of a timing critical net significantly, this could prevent timing goals from being met.

A non-timing critical net may become critical after iterative deletion. If this occurs, the segment assignment of the net is replaced with a segment assignment compatible with the nets initial topology. This process continues until the delay bounds determined for the initial structures are met by the structures after iterative deletion. Therefore, we guarantee that the area optimization performed by iterative deletion will not worsen the circuit performance.

\subsubsection{Edge Selection}

In [16], selection of the maximum cost edge was done by considering the length of an edge, the maximum density of the channel, and the density across the edge. Deletion was done in the most dense regions first, with edge length used to break ties.

We extend the approach through more sophisticated selection of edges for removal. To motivate our new cost function, we first state a number of observations.

For a single channel, we refer to the density for all edges in $S$ as the current density. The channel density for all required edges in $S$ is the required density. Edges than are redundant must be part of some cycle of length $N$ (we select the smallest cycle containing the edge to obtain $N$ ); we define the probable width of such an edge to be $\frac{N-1}{N}$. The probable density is then obtained from the density of all edges considering their probable width. The extension of each of these measures to the variable-width routing model is straightforward.

Our motivation for the use of probable density is as follows. For a long cycle, removal of a single edge can make all other edges on the cycle required, and thus these edges are more likely to exist in the solution obtained after iterative deletion. When the probable density of an area is high, it indicates that density reduction in this area may be difficult; we therefore have a preference for removing edges from this area first.

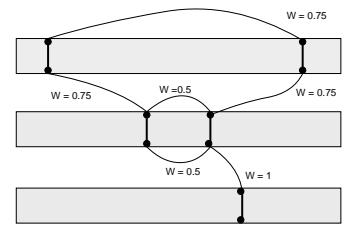

Figure 6. The probable width of an edge is determined by the size of the smallest cycle it belongs to.

We use $e_{\text {required, }} e_{\text {current }}$, and $e_{\text {probable }}$ to denote the various density measures over an edge $\epsilon$. We use $c_{\text {required }}$, $c_{\text {current }}$, and $c_{\text {probable }}$ for the density measures across an entire channel $c$. In general, we will consider an edge $e$ with respect to the channel $c$ that contains it. Given these definitions, we have the following results.

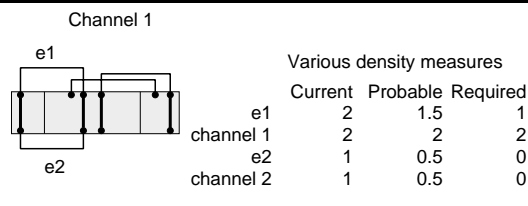

Channel 2

Figure 7. current, required, and probable density measures for edges and channels.

Lemma 1 Required density provides a lower bound on channel density in any connected subset of $S$.

Note that during iterative deletion, required density increases monotonically, while the current density decreases monotonically.

Lemma 2 Edges with current density less than or equal to the channel required density can be part of any optimaldensity subset of $S$.

Proof: Assume we have the optimal solution $O$, which is a subset of the current solution $S$. If we insert all the edges as constrained above, density will not rise above the current density of $S$, which has the required density as a lower bound.

Using these two lemmas, we have the following theorems, which influence our new cost function.

Theorem 1 Given an edge set $S$, and an optimal (nonredundant) edge set $O$, where $O \subseteq S$, and the total density of $O$ is less than that of $S$. There exists edge $e \in S$ in channel $c$ such that $e_{\text {current }}=c_{\text {current }}, e_{\text {current }}>c_{\text {required }}$, and $e \notin O$.

Proof: By contradiction, assume that no such edge exists. Then for each channel, the optimal solution contains channels which either did not have their density reduced ( $\left.c_{\text {current }}=c_{\text {required }}\right)$, or all edges that passed through the 
most dense region of a channel are required in the optimal solution. If this is the case, the total density of $O$ is equal to that of $S$, contradicting the assumption that improvement was possible.

Theorem 2 Given a net $n$, with redundant edge $e$, $e_{\text {current }}>c_{\text {required, }}$, and no other redundant edge $e^{\prime}$ of the same net, where $e_{\text {current }}^{\prime}>c_{\text {required. }}^{\prime}$ Then removal of $e$ from the current solution is compatible with some optimal subset $O$.

Proof: From Lemma 2, we know that even if all remaining edges in $n$ become required, they cannot increase the density of any channel beyond its current required value; thus, they cannot affect the density of some optimal solution $O$. $\square$

Based on these results, the weight of edge $\epsilon$ is

$$
\begin{cases}\text { length }(e) & \text { if } e_{\text {current }} \leq c_{\text {required }} \\ 3 K+\text { length }(e) \times e_{\text {probable }} & \text { if } e_{\text {current }}=c_{\text {current }} \\ 2 K+\text { length }(e) \times e_{\text {probable }} & \text { otherwise }\end{cases}
$$

Edge cost (with $e_{\max }$ as the highest weighted edge other than $e$ in the same cycle) is defined as cost $(e)=2 \times$ weight $(e)-w e i g h t\left(e_{\max }\right)$. We consider the difference between a pair of edges in the same cycle for the following reason: when one edge is removed, the other may become required for net connectivity. Thus, in addition to receiving the benefit of removing the high cost edge, we may also incur a penalty in the other edge becoming required. In our current implementation, we consider the two highest cost edges anywhere in a net, as consideration of specific cycles is computationally expensive.

The large constant $K$, and the ratio between the weights, is used to ensure removal of edges from the most dense regions first (using Theorem 1), and to select edges from nets where the remaining edges cannot increase the required density (using Theorem 2).

\subsection{Summary of the DECIMATE Global Router}

In summary, our global router performs the following steps.

- Interconnect structure selection and optimization for global delay objectives.

- Feedthrough assignment based on consideration of resources and requirements across all rows and nets.

- Iterative deletion to minimize channel density and congestion across the circuit.

- Restoration of net topologies for nets where the structure of a non-critical net has been modified during iterative deletion, and prevents delay objectives from being met.

\section{EXPERIMENTAL RESULTS}

Results of our global router were compared with those of the well known TimberWolf place and route package. This package was previously under development as a university research project $[35,38]$, and is now supported as a commercial product by TimberWolf Systems. We routed a number of MCNC benchmark circuits [25], with placements for these benchmarks being produced by TimberWolfSC. Note that the placements used by the DECIMATE router were obtained after the TimberWolf global router finished: thus, cell mirroring and swapping performed by the TimberWolf global router was included in the placement used by the DECIMATE router. However, all feedthroughs inserted by the TimberWolf global router were removed, and all built-in feedthrough assignments were ignored.

\begin{tabular}{|l||r|}
\hline Benchmark & Improvement \\
\hline fract & $1.4 \%$ \\
\hline struct & $0.7 \%$ \\
\hline primary 1 & $0.2 \%$ \\
\hline primary 2 & $1.9 \%$ \\
\hline biomed & $1.4 \%$ \\
\hline industry 1 & $2.1 \%$ \\
\hline industry 2 & $2.0 \%$ \\
\hline industry 3 & $1.3 \%$ \\
\hline avqsmall & $0.8 \%$ \\
\hline avqlarge & $1.1 \%$ \\
\hline
\end{tabular}

Table 1. Percentage reduction in total tree length of the Steiner heuristic of [6] over minimum spanning tree length for a variety of industry benchmarks.

To motivate our use of minimum spanning trees for interconnect topologies, we first present Table 1, showing the percentage improvement of an algorithm based on the ERT Steiner tree heuristic of [5] over minimum spanning tree length. We consider the existence of equivalent pins, and take advantage of them to reduce tree length. On random examples, we found that the ERT heuristic produced tree lengths approaching that of the 1-Steiner heuristic[23]; the 1-Steiner heuristic obtains improvements of roughly $11 \%$ on large problems. Given pin locations from an actual placement, however, improvements were significantly lower. In this table, we consider wirelength of nets with more than two pins; total wirelength improvements are thus even lower.

Not surprisingly, the slight difference in total tree length results in a slight difference in circuit area. In global routing experiments where we compare minimum spanning tree routing topologies to topologies determined by the ERT heuristic of [5], circuit area results were identical. When we apply the 1-Steiner heuristic of [23], circuit area results are slightly worse, as this heuristic does not take advantage of equivalent pins to reduce tree lengths or minimize feedthroughs.

To demonstrate the effectiveness of the new iterative deletion cost function, we compare density results using our new cost function, iterative deletion with the cost function of [17], and the result of the TimberWolf global router in Table 2. Using feedthrough assignments produced by the TimberWolf global router, the new cost function for iterative deletion resulted in density reductions ranging from $2.5 \%$ to $11.6 \%$. Note that while the iterative deletion process does not employ any form of rip-up and re-route, the density results obtained by the new cost function were only $1.1 \%$ higher on average than the TimberWolf result.

To examine the impact of topology optimization on global routing, we next compare maximum row length, total channel density, and maximum path length performance of the DECIMATE global router, with and without topology optimization, to that of the TimberWolf global router in Table 3. The INDUSTRY benchmarks do not contain signal direction information, so no path length results are reported for these benchmarks. The benchmarks BIOMED, AVQSMALL, and AVQLARGE provided significant improvement in maximum path lengths, with topology optimization providing $52 \%, 32 \%$, and $31 \%$ reductions over the TimberWolf routing result. For deep submicron design, interconnect path lengths can impact signal delay significantly.

Elmore delay performance optimization analysis of the 


\begin{tabular}{|c|c|c|c|c|c|c|c|c|c|}
\hline \multirow[b]{2}{*}{ Benchmark } & \multirow[b]{2}{*}{ Rows } & \multicolumn{2}{|c|}{ TimberWolf } & \multicolumn{3}{|c|}{ DECIMATE } & \multicolumn{3}{|c|}{$\begin{array}{c}\text { DECIMATE + } \\
\text { optimization }\end{array}$} \\
\hline & & L & D & L & D & PL & L & D & PL \\
\hline fract & 6 & 1368 & 39 & 1374 & 41 & 1 & 1374 & 42 & 1 \\
\hline struct & 21 & 4874 & 162 & 4894 & 164 & 0.87 & 4894 & 166 & 0.87 \\
\hline primary 1 & 17 & 4760 & 166 & 4760 & 174 & 1.21 & 4760 & 186 & 0.97 \\
\hline primary 2 & 22 & 10400 & 377 & 10400 & 386 & 0.96 & 10400 & 388 & 0.95 \\
\hline biomed & 44 & 10548 & 728 & 10548 & 536 & 0.58 & 10548 & 535 & 0.48 \\
\hline industry 1 & 17 & 5148 & 449 & 5214 & 433 & - & - & - & - \\
\hline industry 2 & 69 & 14536 & 1006 & 14536 & 1062 & - & - & - & - \\
\hline industry 3 & 52 & 27152 & 1380 & 27152 & 1385 & - & - & - & - \\
\hline avqsmall & 81 & 10272 & 1012 & 10272 & 981 & 0.83 & 10304 & 994 & 0.67 \\
\hline avqlarge & 83 & 10688 & 1009 & 10688 & 1076 & 1.04 & 10608 & 1084 & 0.68 \\
\hline
\end{tabular}

Table 3. Row length (L), total channel density (D), and path length (PL) comparisons with TimberWolf, with and without topology optimization. Path lengths are scaled so that the TimberWolf result is unity.

\begin{tabular}{|l||r|r|r|}
\hline Benchmark & TW & ID $[17]$ & New ID \\
\hline fract & 39 & 40 & 39 \\
\hline struct & 162 & 185 & 164 \\
\hline primary 1 & 166 & 184 & 167 \\
\hline primary 2 & 377 & 412 & 364 \\
\hline biomed & 728 & 729 & 668 \\
\hline industry 1 & 449 & 472 & 447 \\
\hline industry 2 & 1006 & 1107 & 1057 \\
\hline industry 3 & 1380 & 1482 & 1422 \\
\hline avqsmall & 1012 & 1061 & 973 \\
\hline avqlarge & 1009 & 1076 & 993 \\
\hline
\end{tabular}

Table 2. Comparison of cost functions for Iterative Deletion. We show total channel density for an initial TimberWolf placement routing (TW), iterative deletion using the placement and feedthrough assignment of TimberWolf and a simple cost function (ID), and iterative deletion with the improved cost function (New ID).

global routing results is approached as follows. First, as interconnect optimization is most appropriate for deep submicron design, we apply $0.5 \mu$ CMOS IC process parameters of [14], with driver resistance of $2000 \Omega$, and scale the designs accordingly. We assume all interconnect uses the first metal layer, and do not model vias. We assume a cell delay of zero, as this information is not provided with benchmarks for deep submicron design rules. We note again that we consider the static critical path, and only calculate the delay of the interconnect.

For Elmore delay measurements, we did complete experiments on the benchmark BIOMED (we obtained detailed signal information for the other benchmarks only recently, and are in the process of applying $0.5 \mu$ CMOS design rules to them). We show the row width, channel density, maximum path length, and the maximum Elmore delay path (considering only the interconnect) for the TimberWolf routing, a DECIMATE routing without interconnect optimization, a DECIMATE routing with topology optimization, and a DECIMATE routing with both topology optimization and wire sizing in Table 4. We obtain substantial reductions in path lengths and delay, with the bulk of the delay in the TimberWolf solution occurring in two large nets which have circuitous paths. Due to the small size of the circuit and nets, the optimal wiresizing resulted in minimum width interconnections for many segments, and

\begin{tabular}{|l||r|r|r|r|}
\hline & $\mathrm{L}$ & $\mathrm{D}$ & PL & Int. ED \\
\hline TimberWolf & 10548 & 728 & 81932 & $2.925 \mathrm{~ns}$ \\
\hline DECIMATE & 10548 & 536 & 47230 & $2.045 \mathrm{~ns}$ \\
\hline $\begin{array}{l}\text { DECTMATE } \\
\text { + topology }\end{array}$ & 10548 & 535 & 39422 & $1.907 \mathrm{~ns}$ \\
\hline $\begin{array}{l}\text { DECIMATE } \\
\text { + topology } \\
\text { + wire sizing }\end{array}$ & 10548 & 543 & 38566 & $1.899 \mathrm{~ns}$ \\
\hline
\end{tabular}

Table 4. Comparison of Elmore delay results on the BIOMED benchmark: we assume $0.5 \mu$ CMOS IC parameters. We show maximum row length (L), total channel density (D), maximum path length (PL), and Elmore delay of the interconnect (Int. ED).

only limited Elmore delay benefit. Path length differences between the two optimized solutions are a result of different segment assignments (due to the wire sizing) resulting in different path constraints during net restoration. Wire sizing provided limited benefit; for current fabrication technologies, wire sizing may be most appropriate for large nets which can have large drivers, such as clock nets.

In our experiments, we found that interconnect optimization did not necessarily result in a major penalty on circuit area. Relatively few nets required high performance topologies, with the total length of optimized interconnect topologies being quite close to that of the minimum-Steiner tree alternative.

Run times for all benchmarks on a Sun SPARC-10 were modest, ranging from under 1 minute for FRACT to 12 hours for AVQLARGE. The bulk of the run time of our global router is consumed by the iterative deletion process: our edge cost computation routines are quite robust and general, and perform a number of calculations for cost functions we investigated during our research. We expect that a simplification of our global router to support only the specific cost function detailed in this paper will provide a dramatic decrease in run time.

\section{CONCLUSIONS AND FUTURE WORK}

In this paper, we have presented a timing driven global router for standard cell design. It produces area results comparable to or better than that of a current commercial global router, obtains reduced path lengths, lower delay, and does so with relatively low run times and memory consumption. 
We have presented a new problem for performance driven interconnect selection, shown that it is NP-Hard, and have provided a simple heuristic algorithm. We have also introduced the concepts of required and probable density, and have presented a pair of theorems which lead to an improved cost function for the iterative deletion method.

More complex circuit structures may require a more sophisticated approach to interconnect selection; we are currently investigating a variety of methods and delay models. Hill-climbing approaches are an obvious next step, as well as the application of slack-based approaches [32, 19].

A number of extensions are also under way, including support for multi-layer global routing, general cell routing, additional topology and wire sizing algorithms, and more accurate delay modeling.

\section{REFERENCES}

[1] C. J. Alpert, T. C. Hu, J. H. Huang, and A. B. Kahng, "A Direct Combination of the Prim and Dijkstra Constructions for Improved Performance-Driven Routing," Proc. ISCAS, pp. 1869-1872, 1993.

[2] K. Aoshima and E. S. Kuh, "Multi-Channel Optimization in Gate-Array LSI Layout," Proc. ISCAS, 1983, pp. 10051008.

[3] H. B. Bakoglu, Circuits, Interconnections, and Packaging for VLSI, Addison-Wesley, 1990

[4] K. D. Boese, A. B. Kahng, B. A. McCoy, and G. Robins, "Near-Optimal Critical Sink Routing Tree Constructions," IEEE Trans. on Computer-Aided Design of Integrated Circuits and Systems, 14(12), pp. 1417-1436, Dec. 1995.

[5] M. Borah, R. M. Owens, and M. J. Irwin, "An Edge-Based Heuristic for Steiner Routing," IEEE Trans. CAD, no. 13, 1994, pp. 1563-1568.

[6] R. J. Brouwer and P. Banerjee, "PHIGURE: A Parallel Heirarchical Global Router," Proc. 27th DAC, 1990, pp. 650-653.

[7] R. C. Carden IV and C.-K. Cheng, "A Global Router Using an Efficient Approximate Multicommodity Multiterminal Flow Algorithm," Proc. 28th DAC, 1991, pp. 316-321.

[8] C. P. Chen, Y. P. Chen, and D. F. Wong, "Optimal WireSizing Formula Under the Elmore Delay Model," Proc. ACM/IEEE Design Automation Conf., 1996, pp. 487-490.

[9] J. P. Cohoon and L. J. Randall, "Critical Net Routing," Proc. IEEE Int'l. Conf. on Computer Design, pp. 174-177, 1991.

[10] J. Cong, A. B. Kahng, G. Robins, and M. Sarrafzadeh, "Provably Good Performance-Driven Global Routing," IEEE Trans. Computer Aided Design, Vol. 11, No. 6, pp. 739-752, June 1992.

[11] J. Cong and L. He, "Optimal Wiresizing for Interconnects with Multiple Sources," ACM Trans. on Design Automation of Electronic Systems, 1(4), pp. 478-511, Oct. 1996.

[12] J. Cong, L. He, C.-K. Koh, and P. H. Madden, "Interconnect Optimization for High Performance VLSI Design," Integration, j. 21, 1996, pp. 1-94.

[13] J. Cong and K.-S. Leung, "Optimal Wiresizing Under the Distributed Elmore Delay Model," IEEE Trans. on Computer-Aided Design, 14(3), March 1995, pp. 321-336.

[14] J. Cong, K.-S. Leung, and D. Zhou, "PerformanceDriven Interconnect Design Based on Distributed RC Delay Model," Proc. 30th ACM/IEEE DAC, pp. 606-611, 1993.

[15] J. Cong and C.-K. Koh, "Simult aneous Driver and Wire Sizing for Performance and Power Optimization," IEEE Trans. on Very Large Scale Integration (VLSI) Systems, 2(4), December 1994, pp. 408-423.

[16] J. Cong and B. Preas, "A New Algorithm for Standard Cell Global Routing," Proc. of Int. Conf. on Computer-Aided Design, 1988, pp. 176-179.

[17] J. Cong and B. Preas, "A New Algorithm for Standard Cell Global Routing," Integration, j. 14, 1992, pp. 49-65.
[18] W.-M. Dai and E. S. Kuh, "Simultaneous Floor Planning and Global Routing for Hierarchical Building-Block Layout," IEEE Transactions on Computer-Aided Design, vol. CAD-6, no. 5, 1987, pp. 828-837.

[19] J. Frankle, "Iterative and Adaptive Slack Allocation for Performance-driven Layout and FPGA Routing," Proc. ACM/IEEE DAC, pp. 536-542, 1992.

[20] I. Harada and H. Kitazawa, "A Global Router Optimizing Timing and Area for High-Speed Bipolar LSI's," Proc. IEEE $D A C$, pp. 177-181, 1994.

[21] X. Hong, T. Xue, E. S. Kuh, C. K. Cheng, and J. Huang, "Performance-Driven Steiner Tree Algorithms for Global Routing," Proc. ACM/IEEE DAC, pp. 177-181, 1993.

[22] J. Huang, X.-L. Hong, C.-K. Cheng and E. S. Kuh, "An Efficient Timing-Driven Global Routing Algorithm," Proceedings of the 30th Design Automation Conference, 1993, pp. 596-600.

[23] A. B. Kahng and G. Robins, "A New Class of Iterative Steiner Tree Heuristics with Good Performance," IEEE Transactions on CAD 11(7), July 1992, pp. 893-902.

[24] A. B. Kahng and G. Robins, On Optimal Interconnections for VLSI, Kluwer Academic Publishers, 1994.

[25] K. Koźmiński, "Benchmarks for Layout Synthesis - Evolution and Current Status," Proc. Of 28 th Design Automation Conference, 1991, pp. 265-270.

[26] K.-W. Lee and C. Sechen, "A New Global Router for RowBased Layout," Proc. of ICCA D. 1988, pp. 180-183.

[27] J. Lillis, C. K. Cheng and T. T. Y. Lin, "Optimal Wire Sizing and Buffer Insertion for Low Power and a Generalized Delay Model," Proc. IEEE Int'l. Conf. on Computer-Aided Design, Nov. 1995, pp. 138-143.

[28] J. Lillis, C. K. Cheng, T. T. Y. Lin, and C. Y. Ho, "New Performance Driven Routing Techniques With Explicit Area/Delay Tradeoff and Simultaneous Wire Sizing," Proc. ACM/IEEE Design Automation Conf., June 1996 , pp. 395-400.

[29] M. R. Garey and D. S. Johnson, "Computers and Intractability," W. H. Freeman and Co., 1979.

[30] G. Meixner and U. Lauther, "A New Global Router Based on a Flow Model and Linear Assignment," Proc. of Int. Conf. on Computer-Aided Design, 1990, pp. 44-47.

[31] N. Menezes, R. Baldick, and L. T. Pileggi, "A Sequential Quadratic Programming Approach to Concurrent Gate and Wire Sizing," Proc. Int'l Conf. on Computer-Aided Design, 1995, pp. 144-151.

[32] R. Nair, C. L. Berman, P. S. Hauge, and E. J. Yoffa, "Generation of Performance Constraints for Layout," IEEE T. CAD, Vol. 8, No. 8, 1989, pp. 860-874

[33] T. Okamoto and J. Cong, "Buffered Steiner Tree Construction with Wire Sizing for Interconnect Layout Optimization," Proc. Int'l Conf. on Computer-Aided Design, Nov. 1996, pp. 44-49.

[34] T. Okamoto, M. Ishikawa, and T. Fujita, "A New FeedThrough Assignment Algorithm Based on a Flow Model," Proc. ICCAD, Nov. 1993, pp. 775-778.

[35] C. Sechen and A. Sangiovanni-Vincentelli, "TimberWolf3.2: A New Standard Cell Placement and Global Routing Package," Proceedings of the $23 r d$ Design Automation Conference, 1986, pp. 432-439.

[36] E. Shragowitz and S. Keel, "A Global Router Based on a Multicommodity Flow Model," Integration, j. 5, 1987, pp. 3-16.

[37] K. J. Supowit, "Reducing Channel Density in Standard Cell Layout," Proceedings of the 20th Design Automation Conference, 1983, pp. 263-269.

[38] W. Swartz and C. Sechen, "Timing Driven Placement for Large Standard Cell Circuits," Proc. ACM/IEEE DAC, pp. 211-215, 1995. 